\title{
28 Research Square \\ Distribution Of Medical Institutions In Cold Regions Of China Based On Public Health Emergencies
}

\author{
Yue Wu \\ Harbin Institute of Technology \\ Jun $\mathrm{He}$ \\ Harbin Institute of Technology \\ Shanshan Zhang ( $\nabla$ zhangshanshan@hit.edu.cn ) \\ Harbin Institute of Technology
}

\section{Research article}

Keywords: Weighted spatial separation model, Medical Institutions, Distribution, Cold Cities

Posted Date: August 6th, 2020

DOl: https://doi.org/10.21203/rs.3.rs-45000/v1

License: (1) This work is licensed under a Creative Commons Attribution 4.0 International License.

Read Full License 


\section{Abstract}

Background $\mathbb{R}$ Recently, investments in the construction of medical resources have been increasing annually China, and consequently, the allocation of these resources has improved. However, the outbreak of covid-19 in 2020 highlights the problems in the distribution of medical institutions. After the occurrence of public health emergencies, the joint action of different levels of medical and health institutions can bring the role of urban medical and health system into full play. Therefore, after a global public health emergency, the study of medical institution distribution needs to be reconsidered.

Methods $\llbracket$ With the continuous application and development of GIS (Geographic Information System), the application of GIS in civil planning is relatively mature, and research investigating distribution has been conducted in depth. Based on this foundation, this paper analyzes the factors impacting distribution, such as the transportation system, land use characteristics and personal factors, by a weighted spatial separation model of a representative city in a cold region in China.

Results囚The data were sorted, edited and visually processed through the constructed geodatabase to perform an analysis of the spatial distributions of the factors impacting the accessibility of medical institutions in the study area. A weighted spatial separation model was built and applied to comprehensively consider several factors affecting accessibility, the accessibility of these medical institutions is significantly impacted when the spatial population distribution is considered as a factor in the weighted spatial separation model.

Conclusions $\mathbb{X}$ The accessibility of medical institutions in this representative cold city in China was comparatively analyzed in this paper through theoretical research, software computations/simulations and model analysis based on the GIS paradigm. This study will help optimize the layout of medical institutions and improve medical equality.

Trial registration: An ethics review and approval for this study was not required according to the local legislation and institutional requirements.

\section{Background}

Distance is a determining factor of health care facility utility (Pitkin and Varda, 2009); thus, there is a need to minimize the travel time and space distances of health care services delivery (Syed et al., 2013). Spatial accessibility is defined by utilizing a specific method to represent the systematic relationship between the points of departure and destination with consideration of the travel cost (Yang et al., 2016). Improving the efficiency, equality and accessibility of medical and healthcare service systems are significant tasks worldwide. Particularly in cold regions, the decline in driving speed during periods of ice and snow reduces travel efficiency, and the number of traffic conflicts and traffic accidents increases (Munehiro et al., 2011). Thus, the planning and construction of urban medical institutions cannot neglect the locations and spatial relationships in regions with different climates. The rational organization of hierarchical medical services in the geographical space based on traffic efficiency is the basis to ensure 
the high efficiency of medical systems. By improving research on the accessibility of medical institutions in cold regions, fully investigating local situations, and exploring the causes of low accessibility of local medical institutions in combination with geographic information systems (GIS) (Love and Lindquist, 1995), it may be possible to identify how to improve medical services to ensure that basic public health services are equally available to all.

The concept of accessibility was referenced even in early classical location theories, which focused on measuring and evaluating the strengths and weaknesses of locations of specific elements. Early on, Hansen (1959) defined accessibility as the likelihood of interactions between nodes of a transport network. Thereafter, the definition of accessibility was repeatedly extended and improved. After the emergence of GIS, innovative approaches used to measure accessibility based on GIS were widely developed and employed, and various methods are available to identify underserviced or over serviced areas. Most studies use point-based accessibility measurements, including the container, coverage, minimum distance, travel cost, gravity model and two-step floating catchment area (Zhu et al., 2018). The container method measures accessibility based on the number of facilities within a given spatial unit, such as census tract, political district or municipal boundary (Nicholls, 2001). The coverage method, which is also known as the cumulative opportunity method, measures accessibility based on the number of facilities within a certain distance from a demand point (Vaughan et al., 2013). The minimum distance method measures the distance between a demand point and the nearest facility (Smover et al., 2010). The travel cost method measures the average distance between a demand point and all related facilities (Gu et al., 2011). However, most methods disregard the impact of the population distribution on spatial accessibility. Extension produces a spatial interaction metric model that considers the influence of the population distribution on spatial accessibility. The most representative and popular models are the gravity model and the 2SFCA method because the approaches consider both supply and demand. The gravity model calculates an index that is computed by summing the facilities and adjusting for the effect of distance decay (Talen, 2003). The 2SFCA method describes the distance decay effect in a dichotomous manner, namely, people outside the service radius area have no access to the supply facility (Suárez et al., 2011). In most previous studies, accessibility was calculated by these models to measure the travel cost based on the Euclidean distance (Danielsson,1980) instead of the real path distance (Wu et al., 2018). In cold cities, road capacity greatly vary between the winter and summer, and considering measurement results based on the Euclidean distance could result in large errors in accessibility. Therefore, ignoring the difference in travel cost between the winter and other seasons could lead to inaccurate accessibility results of supply facilities. In summary, it is necessary to devote further attention to improving the calculation of travel cost in studies investigating spatial accessibility. The weighted spatial separation model can effectively solve this problem (Zhang, 2014). Accessibility is represented by the average weighted traffic impedance from a starting point to a target point. In the calculation, the calculation result of accessibility is closely related to the scale, attraction and traffic quality between the starting and ending points and is determined by the spatial location of the starting and ending points.

China is a populous country with a rural population of 603 million by 2015 , accounting for $43.9 \%$ (2015 sixth census data of China) of the total population. Since the 1980s, China has carried out reforms in the 
medical insurance system, the prices of medical services and the system of drug production and circulation. However, such medical reform has simultaneously caused problems, such as the unreasonable allocation of health resources and unequal stratification of medical and health services. Furthermore, the current medical treatment system has some problems as follows: the overloading of outpatients at large-scale general hospitals, serious shortage of hospital beds and old facilities in township hospitals and village clinics. In the new medical reform, China has given full attention to the development of small and medium-sized hospitals, which are mentioned in many policy documents, such as "Opinions on Deepening the Reform of the Medical and Health System" and "Deepening the System of Medical and Health Care in 2009-2011" (the State Council of the People's Republic of China). Both documents were reviewed and approved by the State Council's executive committee and referred to the need to "Improve the primary health care service system, with a focus on strengthening the construction of county-level hospitals (including Chinese medicine hospitals), township hospitals, village clinics in remote areas and urban community health service centers in difficult areas". Currently, the most scientific and effective method is to make the best use of the existing medical resources under the unified regulation and control of the government, adjust the distribution of medical resources with priorities and goals, and establish a hierarchical medical and health network structure from top to bottom. To address the issue, some researchers have proposed various extensions of point-based accessibility measurements to optimize the planning and construction of medical resources. Knox (Knox, 1980) used isolines to visualize the spatial layout of medical facilities at Edinburgh in terms of accessibility. Kilinc et al. (2016) studied the potential spatial accessibility of family care services and revealed differences in the accessibility of different family care services by analyzing factors impacting the supply-demand relationships of medical resources for family care in the state of Arkansas both within geographical regions and between regions. Smith et al. (2017) described a methodologically accessible optimal combination algorithm to improve the drafting of service strategies and cut costs. By considering a case study of tuberculosis clinics in London, they generated a service portfolio with optimal spatial accessibility on the premise of rationalized services. Mizen et al. (2015) compared several algorithms for calculating the accessibility of medical and health institutions. Their comparative analysis suggested that calculating the accessibility of both urban and rural medical institutions in the same way leads to errors. They explored the causes of these errors, quantified them and presented recommendations for calculation procedures. Stentzel et al. (2018) measured the feasibility of medical and healthcare services in rural areas with low population density in West Pomerania in northern Germany based on GIS network analysis functions. They analyzed whether the accessibility of general doctors and gynecologists depended on the time spent traveling by car or public transport. Yeeun et al. (2018) developed an enhanced algorithm for calculating the accessibility of medical institutions in Seoul, Korea, with the aim of guaranteeing accurate accessibility calculations for medical institutions in this city, which has a high population density and numerous and densely distributed hospitals.

In the above studies, the allocation, planning and layout of medical institutions were examined along with the division of functional spaces and the organization of traffic flow lines. However, studies investigating cold regions remain lacking, although the general accessibility of medical institutions has been 
investigated in depth. For similar studies of the accessibility of medical institutions in representative cold cities in China, it may be necessary to apply distinct approaches to provide adequate theoretical support for analyzing different regions with varying conditions throughout the country. Therefore, this paper aims to analyze the spatial coverage of medical institutions, the accessibility of medical institutions' services, the accessibility in the corresponding vector data network, and the accessibility in a weighted spatial separation model, analysis and counting based on a geodatabase built for a representative cold city in China. The second section of this paper presents the methodology of this study and introduces the current situation in the study area and the weighted spatial separation model. In the third section, Harbin, which is a representative cold city in China, is studied as the research object; the basic spatial data, including spatial road and medical facilities and population distribution, are analyzed using a spatial separation model. Then, a weighted spatial separation model was built considering separation factors, including the spatial coverage separation, the service areas separation, the road network separation, the population separation and the weather separation. Finally, the accessibility of medical institutions in the study area is explored by analyzing data regarding the related separation factors.

\section{Method}

\subsection{Case Study}

The term "cold cities", in general, refers to cities where winter lasts for a rather long period and weather conditions are harsh, thus exerting adverse impacts on urban life. In such a city, the average atmospheric temperature is usually below $-10^{\circ} \mathrm{Cin}$ January. Such cities are generally known as "cold cities" in China, "winter cities" in Europe and America, and "northern cities" in Japan. In this paper, Harbin, a city in Heilongjiang, China, which is a representative cold city, is chosen as the research object. The spatial distributions of basic resources and the accessibility of medical institutions are studied. The general situation in the study area is summarized in Table 1.

Table 1. General Situation of the Representative Cold City in China

\begin{tabular}{ll}
\hline Summary of the Study Area & Harbin, Heilongjiang, China \\
\hline Latitude & $45^{\circ} 64^{\prime} \mathrm{N}-45^{\circ} 86^{\prime} \mathrm{N}$ \\
Longitude & $126^{\circ} 48^{\prime} \mathrm{E}-126^{\circ} 84^{\prime} \mathrm{E}$ \\
Climatic Features & Temperate continental climate \\
Size of the Study Area & $591 \mathrm{~km}^{2}$ \\
Catchment Area & $69 \mathrm{~km}^{2}$ \\
Land Area & $522 \mathrm{~km}^{2}$ \\
Population Size & $3,959,100$ \\
Research Scope & Within beltways \\
\hline
\end{tabular}

In this paper, medical institutions are classified into secondary and tertiary general hospitals and community healthcare centers. There are 36 secondary and higher-level general hospitals (including 12 tertiary general hospitals, 4 tertiary traditional Chinese medicine hospitals, 3 tertiary military hospitals and 17 secondary general hospitals) and 75 community healthcare centers in the study area. With an area of $591 \mathrm{~km}^{2}$ and a population of 3,959,200, Harbin consists of five district-level administrative divisions 
(including the districts of Daoli, Nangang, Daowai, and Songbei) and 90 township-/street-level administrative divisions (including Jianguo Street, Gongcheng Street and Chengxiang Road).

\subsection{Weighted Spatial Separation Model}

The spatial separation model is an accessibility measurement model widely used in traffic network research. The model formula is as follows:(see Equation 1 in the Supplemental Files)

Where is the accessibility of staring point $i$. The accessibility of starting point $i$ is expressed by calculating the traffic impedance from starting point I to target point $i$, which is usually defined as the space distance, time consumption or traffic cost between $i$ and $j$. Consider the spatial barrier a factor to measure the accessibility of medical institutions could significantly affect the travel cost as shown in figure 1. The range of the red circle represents the walking distance of the medical facility based on the Euclidean distance, while the range circled by the black line considers the walking distance of the space barrier, and the range of accessibility clearly greatly varies.

However, when calculating accessibility between the starting and target points, this model treats all spatial nodes the same and lacks other accessibility factors, such as attraction between the two points and the nature of land use, and only calculates accessibility from a single dimension. The weighted spatial separation model selected in this study is a measurement model that comprehensively considers a variety of factors and is a measurement model commonly used in complex accessibility calculations. Accessibility in a model of weighted spatial separation can be measured based on the accessibility of the corresponding road vector network. This approach provides a means of considering spatial interactions with weights to comprehensively consider the factors impacting accessibility. The process of calculating accessibility using a weighted spatial separation model is rather complicated. However, the calculation results are reasonably consistent with the practical situation. In addition to the spatial distributions of the road network and the medical institutions, the spatial population distribution is also considered in the weighted spatial separation model used in this study. The calculations were performed as follows. First, the township- and street-level administrative divisions were statistically analyzed based on the measured vector data network, and the accessibilities were measured via normalization. Then, a suitable calculation model was selected after an analysis and comparison. To accurately calculate the spatial accessibilities of the medical institutions in each block, the formulas were optimized to calculate the weighted spatial separation model. By normalizing the vector data and traffic accessibility, the accessibilities of the medical institutions nearest to each block (including both community healthcare centers and general hospitals) were determined based on the weighted average traffic impedance. The lower the calculated model result, the closer the connection to the target point, and the higher the accessibility. (see Equation 2 in the Supplemental Files)

where $A_{i}$ is the accessibility of a block, $d_{i j}$ is the spatial impedance (the shortest time) of traveling from block $i$ to the nearest general hospital $j, D_{i}$ is the degree of need (the population size) at target point $i$, and $n$ is the total number of nodes in the evaluated system. 


\section{Results}

The data of statistic analysis include the roads, medical institutions and urban population of Harbin were acquired from data collections and field surveys [1]. The data were sorted, edited and visually processed through the constructed geodatabase to perform a analysis of the spatial distributions of the factors impacting the accessibility of medical institutions in the study area.

\subsection{Spatial road distribution}

After the obtained road network data were cleaned and processed, the roads of Harbin were divided into four levels. Using the Jenks natural breaks classification method, the roads were divided into five density levels, including low density, moderately low density, moderate density, moderately high density, and high density, as shown in Fig 2b.From the visualized output from the statistical analysis of the road network data, it can be seen that the spatial distribution of the road network in Harbin is not uniform, nor are the densities of these roads; the ratio of low-density to high-density roads is 24 to 1 .

By measuring the time costs, the accessibilities of the nearest community healthcare centers and the nearest general hospitals were forecast. These accessibilities were graphically represented by means of spatial interpolation and inverse distance weighting. The vector data network was classified into nine accessibility categories $(1,2,3,4,5,6,7,8$ and 9$)$ by dividing the measurements into equal intervals as shown in Fig 3. The accessibility south of the Songhua River is much higher than that to the north and is the highest in downtown areas within the second ring. In the three areas north of the Songhua River, the accessibility of the nearest community healthcare centers is relatively high, whereas the accessibility of the nearest general hospitals is relatively low.

\subsection{Spatial distribution of medical facilities}

The distribution of medical institutions has diffused outwards from the city center. In this city, general hospitals are mostly located in downtown areas, and community healthcare centers are also most densely distributed in downtown areas as shown in Fig 4.

The spatial coverage of medical institutions can be represented in the form of buffers around the institutions. To construct the buffers of medical institutions, it can be assumed that services are available within 2,000 $\mathrm{m}$ when traveling by foot and 5,000 $\mathrm{m}$ when driving. Accordingly, 5,000 m, 3,500 m and 2,000 m buffers were established for the tertiary general hospitals, secondary general hospitals and community healthcare centers of Harbin as shown in Fig 5. The service areas of the tertiary general hospitals, secondary general hospitals and community healthcare centers are $310.93 \mathrm{~km}^{2}, 186.82 \mathrm{~km}^{2}$ and $310.93 \mathrm{~km}^{2}$, respectively, and the total service coverage is $64.12 \%$. Nangang district has the highest service coverage, which is under $97.17 \%$ with a $86.50 \mathrm{~km}^{2}$ coverage area. Xiangfang district has the poorest conditions with service coverage of $46.04 \%$ and a coverage area of $76.59 \mathrm{~km}^{2}$. Services from 
general hospitals cover most areas of urban districts. There are 36 general hospitals in the study area, but most of their service buffers spatially overlap. The spatial distribution of medical resources is denser within the second ring, whereas there are fewer medical institutions outside the second ring. In addition, there are far more medical resources available south of the Songhua River than north. Community healthcare centers cover most street-level administrative divisions and almost all streets in downtown areas. However, the street-level administrative divisions are larger outside the second ring.

The service areas of medical institutions can be represented by buffer zones and Voronoi diagrams obtained through superimposition as shown in Fig 6. Notably, the service areas of community healthcare centers and general hospitals are $272.00 \mathrm{~km}^{2}$ and $315.20 \mathrm{~km}^{2}$. The natural breaks classification method was used to classify the accessibility of the community healthcare centers and general hospitals according to the spatial density of the road network within each service area. The accessibility was classified into five levels, namely, high, moderately high, moderate, moderately low and low. The results suggest that the accessibility of the service areas of both community healthcare centers and general hospitals is very good; their overall accessibility levels are $81.63 \%$ and $86.77 \%$. Hence, these medical institutions and hospitals are sufficient to meet most daily needs with regard to medical treatment as shown in Table 2.

Table 2. Accessibility of Services from Medical Institutions in Harbin

\begin{tabular}{|c|c|c|c|c|}
\hline \multirow[t]{2}{*}{$\begin{array}{l}\text { Accessibility } \\
\text { Level }\end{array}$} & \multicolumn{2}{|c|}{$\begin{array}{l}\text { Accessibility of Services from Community } \\
\text { Healthcare Centers }\end{array}$} & \multicolumn{2}{|c|}{$\begin{array}{c}\text { Accessibility of Services from General } \\
\text { Hospitals }\end{array}$} \\
\hline & Coverage & Percentage & Coverage & Percentage \\
\hline High & $119.15 \mathrm{~km}^{2}$ & $43.81 \%$ & $140.57 \mathrm{~km}^{2}$ & $44.60 \%$ \\
\hline $\begin{array}{l}\text { Moderately } \\
\text { High }\end{array}$ & $85.05 \mathrm{~km}^{2}$ & $31.27 \%$ & $101.12 \mathrm{~km}^{2}$ & $32.08 \%$ \\
\hline Moderate & $17.82 \mathrm{~km}^{2}$ & $6.55 \%$ & $31.79 \mathrm{~km}^{2}$ & $10.09 \%$ \\
\hline $\begin{array}{l}\text { Moderately } \\
\text { Low }\end{array}$ & $37.46 \mathrm{~km}^{2}$ & $13.77 \%$ & $25.56 \mathrm{~km}^{2}$ & $8.11 \%$ \\
\hline Low & $12.52 \mathrm{~km}^{2}$ & $4.60 \%$ & $16.16 \mathrm{~km}^{2}$ & $5.13 \%$ \\
\hline
\end{tabular}

\subsection{Spatial population distribution}

Data on the administrative boundaries of the 90 township-/street-level divisions within Harbin were combined with data from the sixth population census of China to calculate the population density. Using the Jenks method, the population density was divided into five levels, namely, $12-5,871$ residents $/ \mathrm{km}^{2}$, $5,872-18,424$ residents $/ \mathrm{km}^{2}, 18,425-37,315$ residents $/ \mathrm{km}^{2}, 37,316-57,147$ residents $/ \mathrm{km}^{2}$, and 57,14899,883 residents $/ \mathrm{km}^{2}$, as shown in Fig 7 .

The population distribution in Harbin is more concentrated at the city center, and edge effects are significant. The major cause of this phenomenon lies in the relatively short development history of Harbin. As a city expands, residents and industries tend to transfer to the suburbs. However, the population density remains higher within main urban areas. As time passes, the city gradually develops, and the population density gradually declines in the city center. The gradient from low density to high 
density decreases, and the city becomes decentralized. Likewise, the population density will become relatively steady with the increase in income, enlargement of the city scale, and reduction of transportation costs within the city.

\section{Discussion}

In previous studies, the service radius was considered a key factor to measuring the reach of medical facilities on the premise that there was no barrier between the starting point and the destination. However, in the actual path affected by road, nature and other aspects of barrier factors, no barrier can be reached simultaneously. Therefore, barrier factors affecting the accessibility of medical institutions need to be discussed. Distance and travel cost are the two most basic quantitative indicators affecting accessibility. Barrier factors are analyzed from these two aspects. Among the factors, distance is most commonly affected by road network barriers, spatial coverage and service area of medical institutions, while weather barriers restrict the travel cost of people to arrive at medical institutions.

Based on the above analysis, the degree of need is affected by multiple factors, including the spatial coverage, service areas, road network and population distribution; thus, $D_{i}=h\left(C_{i}+S_{i}+R_{i}+P_{i}+W_{i}\right)$, where $C i$ is the spatial coverage separation, $S_{i}$ is the service areas separation, $R_{i}$ is the road network separation, $P_{i}$ is the population separation and $W_{i}$ is the weather separation. The formula of the weighted spatial separation model is as follows (see Equation 3 in the Supplemental Files)

All factors were substituted into the corresponding formula to obtain the weighted spatial separation model, and the results were statistically analyzed and visualized as shown in Fig 8.

As seen from the visualized outputs, the accessibilities of community healthcare centers and general hospitals are mostly high or moderately high in the downtown areas of Harbin but low or moderately low to the north of the Songhua River and in the outskirts of the city. The statistical data analysis suggests that in Harbin, the accessibility of general hospitals is much higher than that of community healthcare centers. The accessibility of general hospitals is $3.97 \%, 1.15 \%$ and $4.31 \%$ higher than that of community healthcare centers for institutions of high, moderately high and moderate accessibility, respectively. Meanwhile, community healthcare centers and general hospitals with low and moderately low accessibility account for a considerable proportion (59.18\% and $49.75 \%$, respectively) of all institutions as shown in Fig. 9.

When the spatial population distribution is not considered, the spatial distribution and accessibility of the medical institutions in Harbin are centralized and high in the downtown areas. In regions farther from downtown, the spatial distribution is more decentralized, and the accessibility is lower. However, the accessibility of these medical institutions is significantly impacted when the spatial population distribution is considered as a factor in the weighted spatial separation model, on the grounds that the population size directly impacts the need for medical services. In cases of great need for such services, traffic congestion or a shortage of medical resources will arise. In this case, medical institutions will 
become less accessible. In Harbin, the population density, medical institutions and roads are all concentrated in downtown areas and are scarce on the outskirts of the city. When the spatial distributions of the population density, medical institutions and road network are all taken into account as factors impacting accessibility when calculating the model weights, the accessibility is found to be either high or low in areas where the distributions are concentrated because of how the different factors interact with and restrict each other. However, the accessibility is relatively low in most areas where the densities of the population, medical institutions and road network are low.

\section{Conclusion}

In this paper, problems related to the accessibility of medical institutions in a representative cold city in China were studied via GIS analysis. Accessibility was measured by analyzing the traffic impedance between different start and end points and considering the difficulty of reaching the target point due to this impedance. In this study, a geodatabase was constructed for a representative cold city (the study area) in China by integrating GIS techniques with accessibility research using a weighted spatial separation model. Factors impacting accessibility, including the transportation system, land use characteristics and personal factors, were analyzed. A weighted spatial separation model was built and applied to comprehensively consider several factors affecting accessibility, including the spatial coverage separation, the service areas separation, the road network separation, the population separation and the weather separation. To calculate the accessibility of medical institutions using a weighted spatial separation model, Harbin was chosen as a case study. The accessibility of medical institutions was analyzed.

Although the accessibility of medical institutions in this representative cold city in China was comparatively analyzed in this paper through theoretical research, software computations/simulations and model analysis based on the GIS paradigm, this study still has many limitations and shortcomings. On the one hand, many differences exist among different areas of China, especially cultural and economic differences, although the planning of Harbin can be considered truly representative of cold regions in China. On the other hand, this study was performed on the premise that residents require the services of public medical institutions. Only general hospitals and community healthcare centers were studied. In addition, the spatial distribution of the medical institutions was evaluated only on the basis of the availability of medical services. Consequently, the research findings might be not consistent with the actual availability of medical facilities and services. In this case, the measured accessibility of medical institutions might be inaccurate, and residents' actual needs for medical services may not be properly accounted for. Last but not least, the spatial data used in this study were acquired from open-source maps available on the Internet. Over time, such data on traffic, roads and transportation modes will change. Furthermore, it is unknown whether the trends may change in certain areas, and such changes would impact the findings of this study to some extent. Therefore, the applicability of this study is subject to certain temporal limits with regard to the calculations and statistics concerning the accessibility of medical institutions in China. 


\section{Abbreviations}

GIS (Geographic Information System)

\section{Declarations}

\section{Funding}

The work is financially supported by the National Natural Science Foundation of China (NSFC) (51808160) and the Fundamental Research Funds for the Central Universities (HIT.NSRIF.2020035).

\section{Source}

All the figures are drawn by the author $\mathrm{YW}$ and $\mathrm{JH}$.

Ethics approval and consent to participate: Not applicable. An ethics review and approval for this study was not required according to the local legislation and institutional requirements.

Consent for publication: Written informed consent for publication was obtained from all participants.

Availability of data and material: Not applicable.

Competing interests: The authors declare that the research was conducted in the absence of any commercial or financial relationships that could be construed as a potential conflict of interest.

Authors' contributions: Author Contributions: Conceptualization, Y.W., J.H., SS.Z.; Methodology, Y.W., J.H., SS.Z.; Investigation, Y.W.; Formal Analysis, Y.W.; Data Curation, J.H.; Writing-Original Draft Preparation, Y.W.; Writing-Review and Editing, Y.W., J.H., SS.Z..; Visualization, Y.W.; Supervision, SS.Z.; Project Administration, SS.Z.; Funding Acquisition, Y.W.

Acknowledgements: The authors want to thank the local government of Harbin, China for their permission for data providing and the permission of investigation.

\section{References}

[1] Pitkin, D.K., Varda, D.M. (2009) Social capital and health care access: A systematic review. Med. Care Res. Rev.,66: 272-306.

[2] Syed, S.T., Gerber, B.S., Sharp, L.K. (2013) Traveling towards disease: Transportation barriers to health care access.J. Community Health, 38: 976-993.

[3] Yang, N., Chen, S., Hu, W., Wu, Z., Yi, C. (2016) Spatial Distribution Balance Analysis of Hospitals in Wuhan. Int. J. Environ. Res. Public Health, 13: 971. 
[4] Munehiro, K., Takemoto, A.,Kasai, S., Asano, M. (2011) Analysis on rural highway design using traffic micro-simulation in cold regions. Procedia - Social and Behavioral Sciences,16: 388-399.

[5] Love, D., Lindquist, P. (1995) The geographical accessibility of hospitals to the aged: a geographic information systems analysis within Illinois. Health Services Research, 29(6): 629.

[6] Hansen W. G. (1959) How accessibility shapes land-use. Journal of the American Institute of Planners, 25(2): 73-76.

[7] Zhu X.M., Tong, Z.J., Liu, X.P., Li, X.Q., Lin, P.D., Wang, T. (2018) An Improved Two-Step Floating Catchment Area Method for Evaluating Spatial Accessibility to Urban Emergency Shelters. Sustainability, 10(7): 2180-2194.

[8] Nicholls, S. (2001) Measuring the accessibility and equity of public parks: A case study using GIS. Manag. Leis, 6: 201-219.

[9] Vaughan, K.B., Kaczynski, A.T., Wilhelm, S.S.A., Besenyi, G.M., Bergstrom, R., Heinrich, K.M. (2013) Exploring the distribution of park availability, features, and quality across Kansas city, Missouri by income and race/ethnicity: An environmental justice investigation. Ann. Behav. Med., 45 (Suppl. 1): S28-S38.

[10] Smoyer-Tomic, K.E., Hewko, J.N., Hodgson, M.J. (2010) Spatial accessibility and equity of playgrounds in edmonton, canada. Can. Geogr., 48: 287-302.

[11] Gu, Z., Xu, W., Yuan, Y., Zhou, H., Ge, Y. (2011) Evaluation of Disaster Shelter Planning in Rural Areas: A Case Study of Xiaoyudong Town in Sichuan Province. J. Catastrophol, 26: 115-119. (In Chinese)

[12] Talen, E. (2003) Neighborhoods as service providers: A methodology for evaluating pedestrian access. Environ. Plan. B Plan. Des, 30: 181-200.

[13] Suárez-Vega, R., Santos-Peñate, D.R., Dorta-González, P., Rodríguez-Díaz, M. (2011) A multi-criteria GIS based procedure to solve a network competitive location problem. Appl. Geogr, 31: 282-291.

[14] Danielsson P E. (1980) Euclidean distance mapping. Computer Graphics \& Image Processing, 14(3):227-248.

[15] Wu, H., Liu, L., Yu, Y., Peng, Z. (2018) Evaluation and Planning of Urban Green Space Distribution Based on Mobile Phone Data and Two-Step Floating Catchment Area Method. Sustainability, 10, 214.

[16] Zhang, Q. (2014) Accessibility evaluation of the primary school pedestrian network under space separation. University of Chongqing, China.

[17] 2015 sixth census data of China. National bureau of Statistic of China.

[18] Opinions on Deepening the Reform of the Medical and Health System,the State Council of the People's Republic of China, 2016. 
[19] Deepening the System of Medical and Health Care in 2009-2011,the State Council of the People's Republic of China, 2009.

[20] Knox, P. L. (1980) Measures of accessibility as social indicators: a note. Social Indicators Research, 7(1-4): 367-377.

[21] Kilinc, M. S., Milburn, A. B., Heier Stamm, J. L. (2016) Measuring potential spatial accessibility of home healthcare services. Socio-Economic Planning Sciences, , 09: 1-7.

[22] Smith, C. M., Fry, H., Anderson, C., Maguire, H., Hayward, A. C. (2017) Optimising spatial accessibility to inform rationalisation of specialist health services. International Journal of Health Geographics, 16(1): 15.

[23] Mizen, A., Fry, R., Grinnell, D., Rodgers, S. E. (2015) Quantifying the error associated with alternative gis-based techniques to measure access to health care services. Aims Public Health, 2(4): 746-761.

[24] Stentzel, U., Bahr, J., Fredrich, D., Piegsa, J., Hoffmann, W., Berg, N. V. D. (2018) Is there an association between spatial accessibility of outpatient care and utilization? analysis of gynecological and general care. Bmc Health Services Research, 18(1): 322.

[25] Yeeun, K., Young-Ji, B., Hwasoo, Y., Ikram, S. T. (2018) Enhancing healthcare accessibility measurements using gis: a case study in seoul, korea. PLOS ONE, 13(2): 1-19.

[26] City of Harbin. City of Harbin Open Data Portal. Available online: URL http://data.harbin.gov.cn/.

\section{Figures}


+ medical facility

$\rightarrow 10$ mins walking with road network barrier

- 10 mins walking without road network barrier

- 5 mins walking without road network barrier

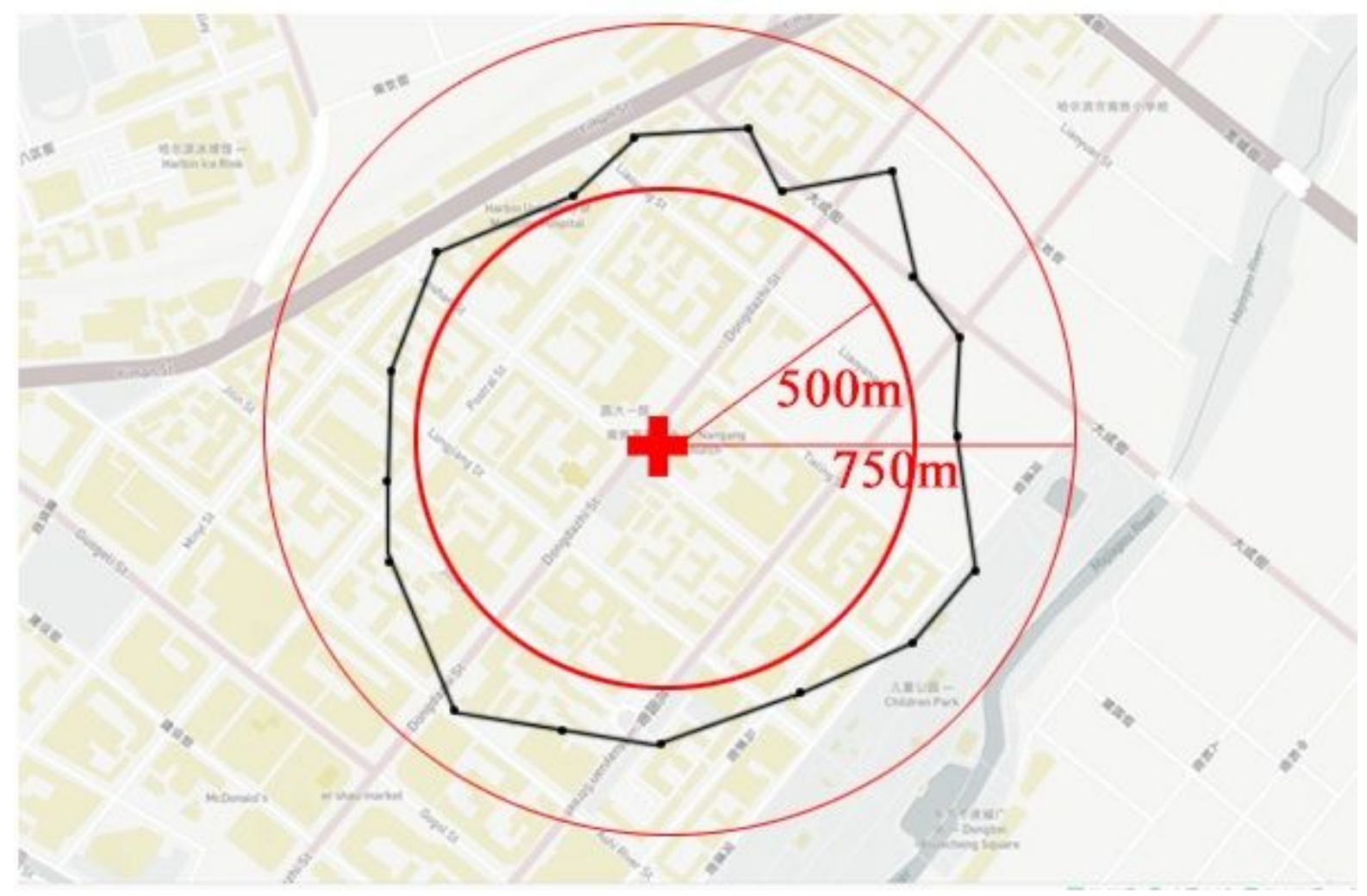

Figure 1

Scope of 10-minute walking distance from the medical facility with or without a road network barrier 


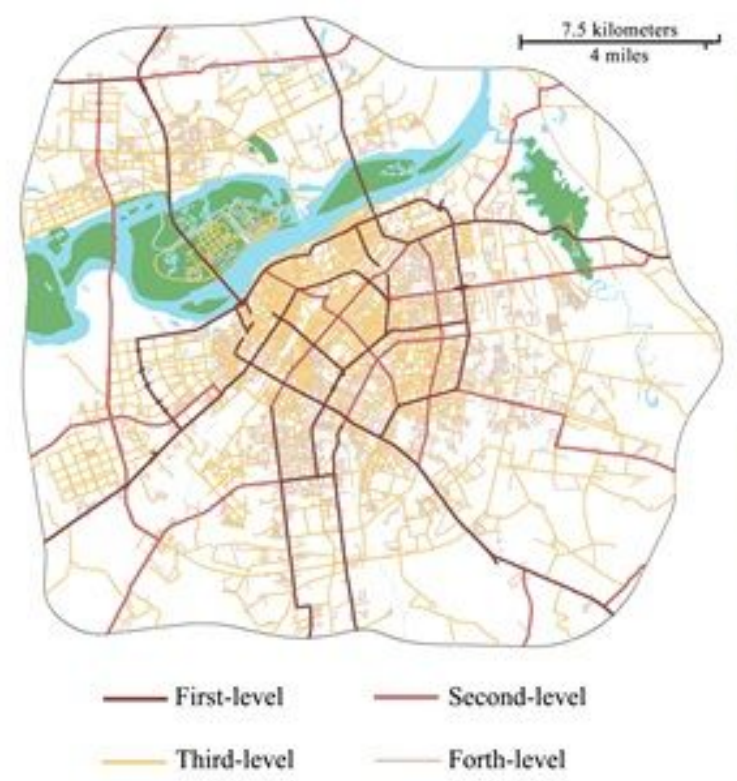

a Distribution of Roads at Varying Levels

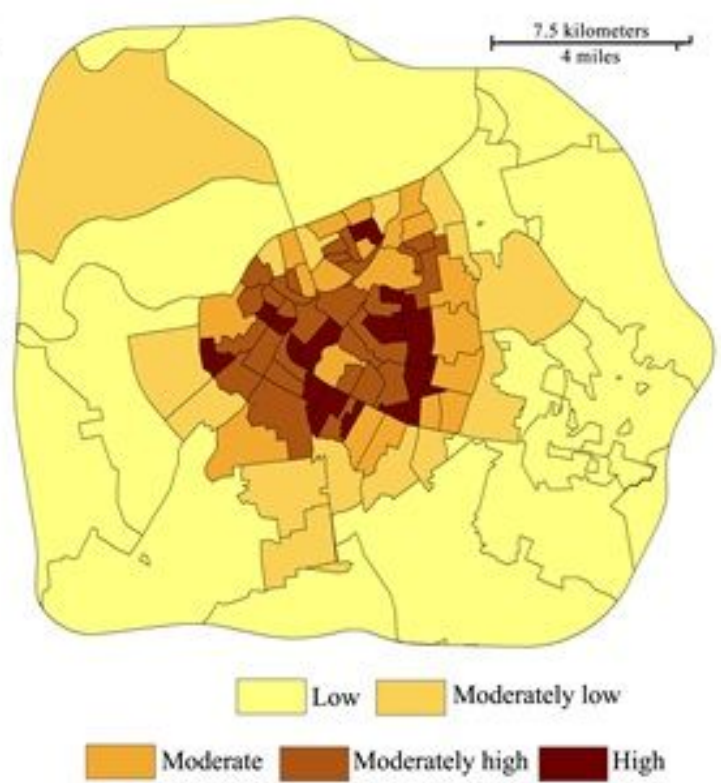

b Density Distribution of Roads

\section{Figure 2}

Spatial Distribution of Roads in Harbin
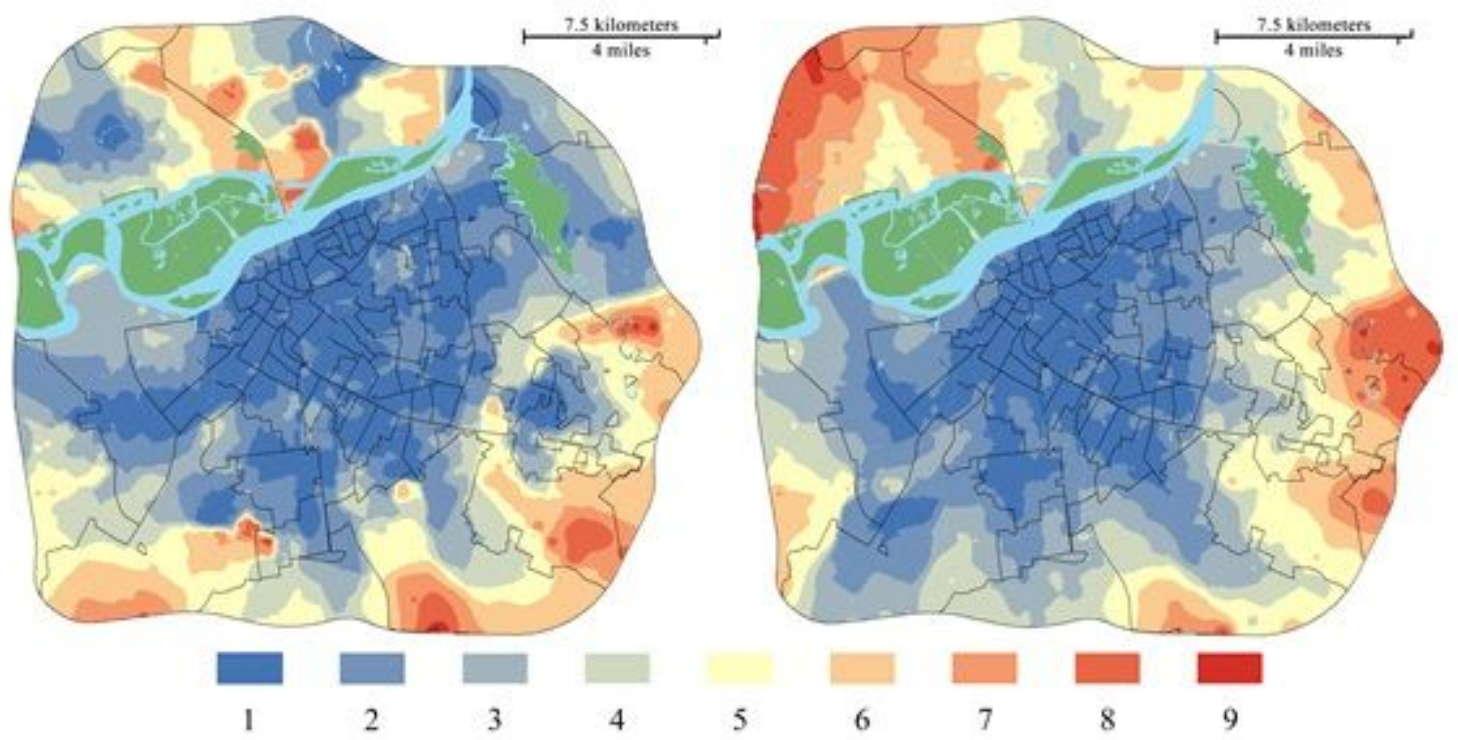

a Accessibilities of the Nearest Community Healthcare Centers b Accessibilities of the Nearest General Hospitals

\section{Figure 3}

Accessibilities of the Nearest Medical Institutions in Harbin 


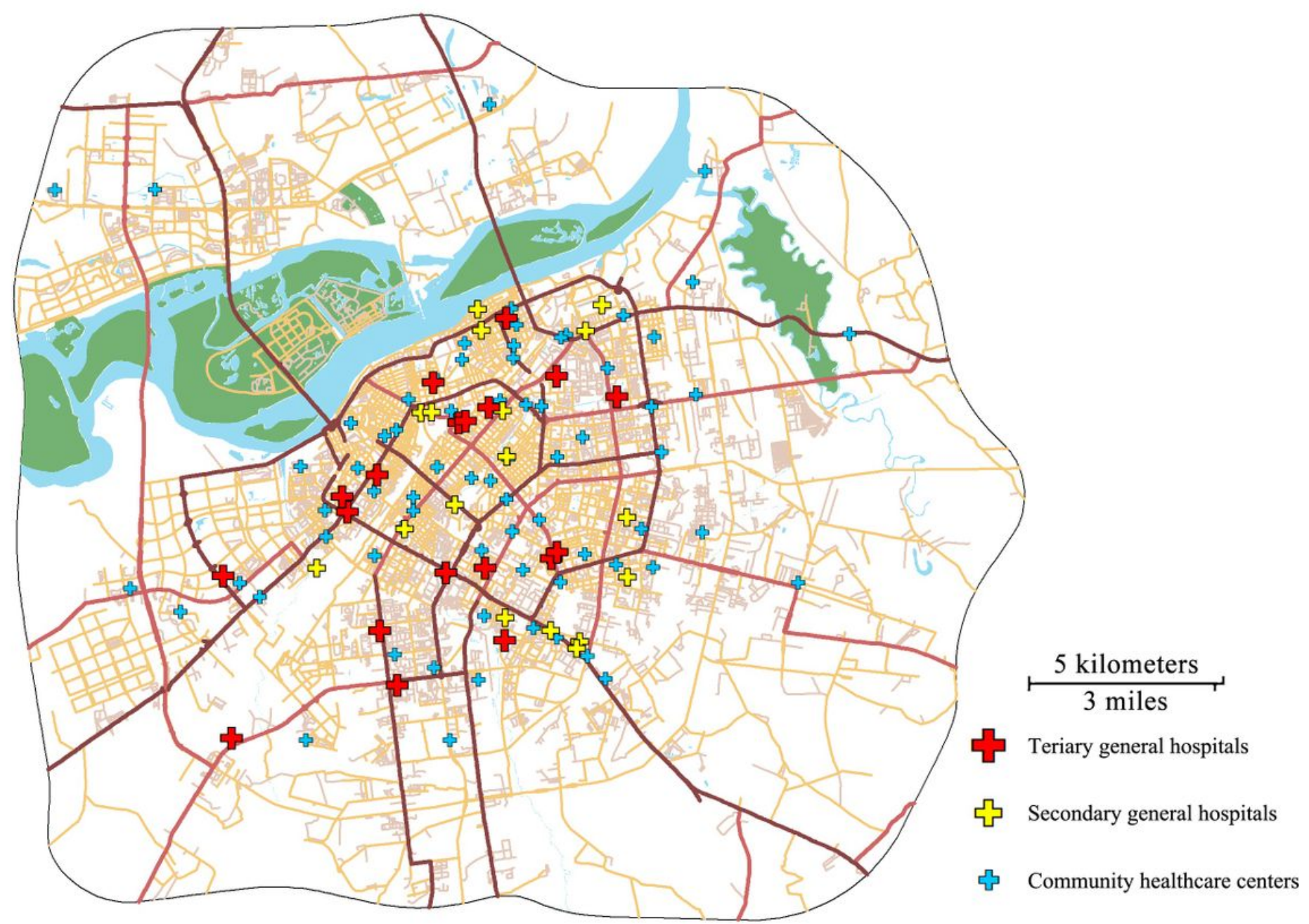

Figure 4

Spatial Distribution of Medical Institutions in Harbin 


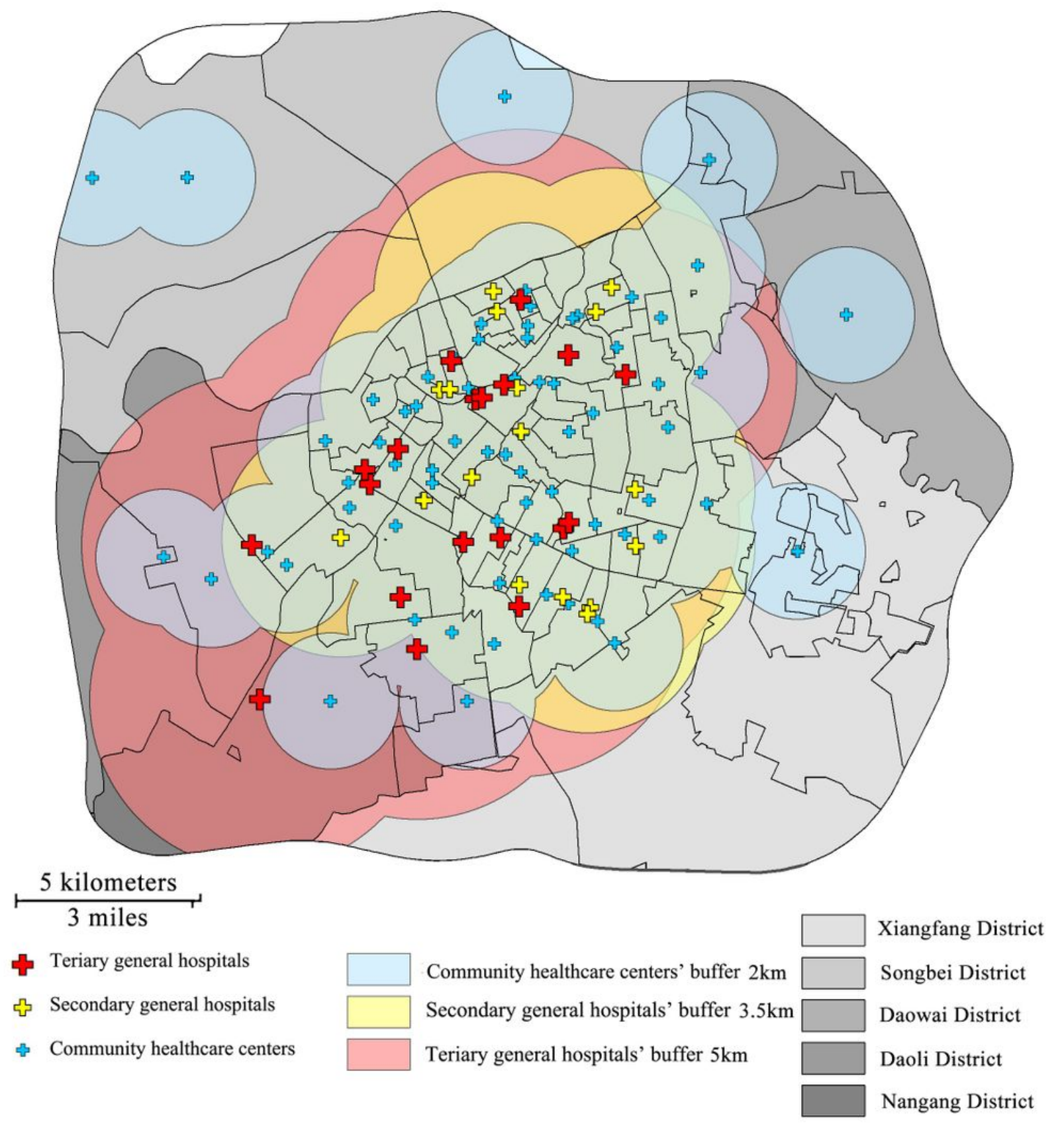

Figure 5

Buffers of Medical Institutions in Harbin 

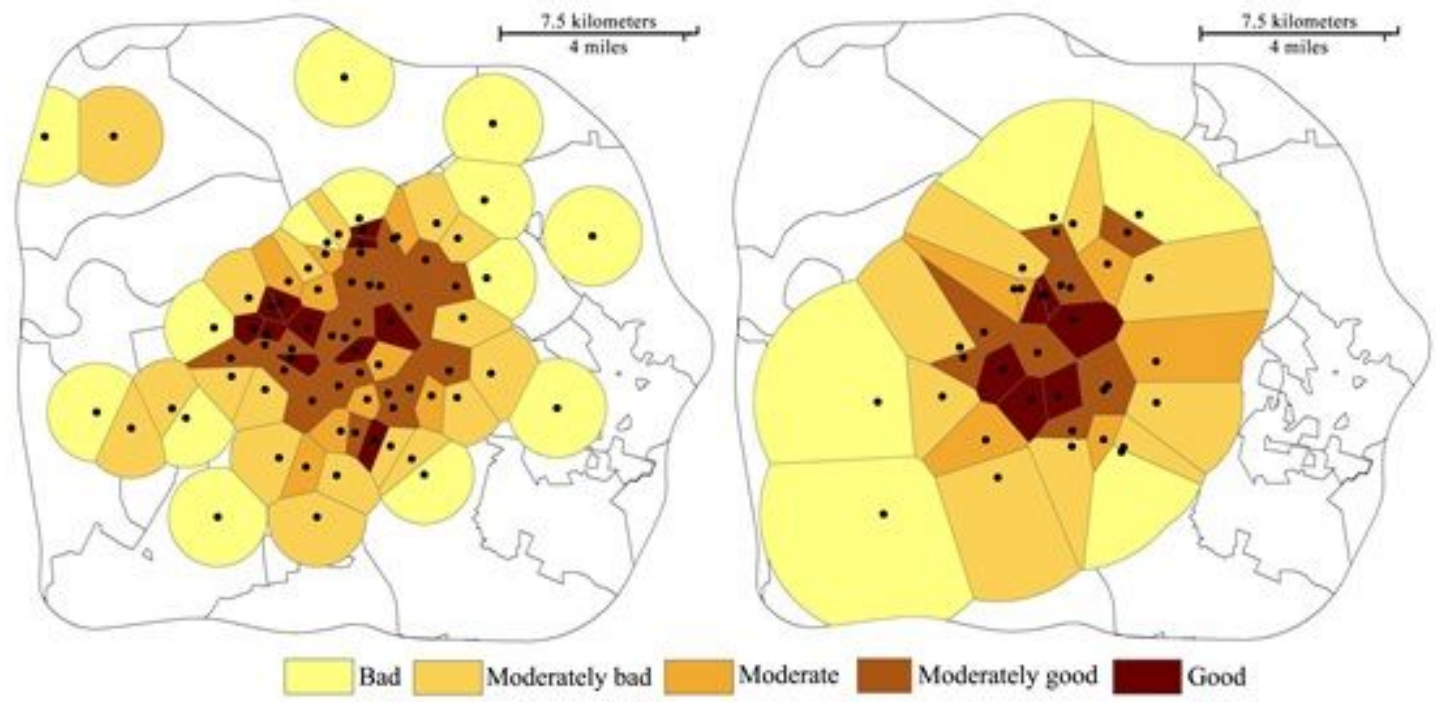

a Accessibility of Community Healthcare Center Service Areas in Harbin b Accessibility of General Hospital Service Areas in Harbin

\section{Figure 6}

Accessibility of Service Areas of Medical Institutions in Harbin

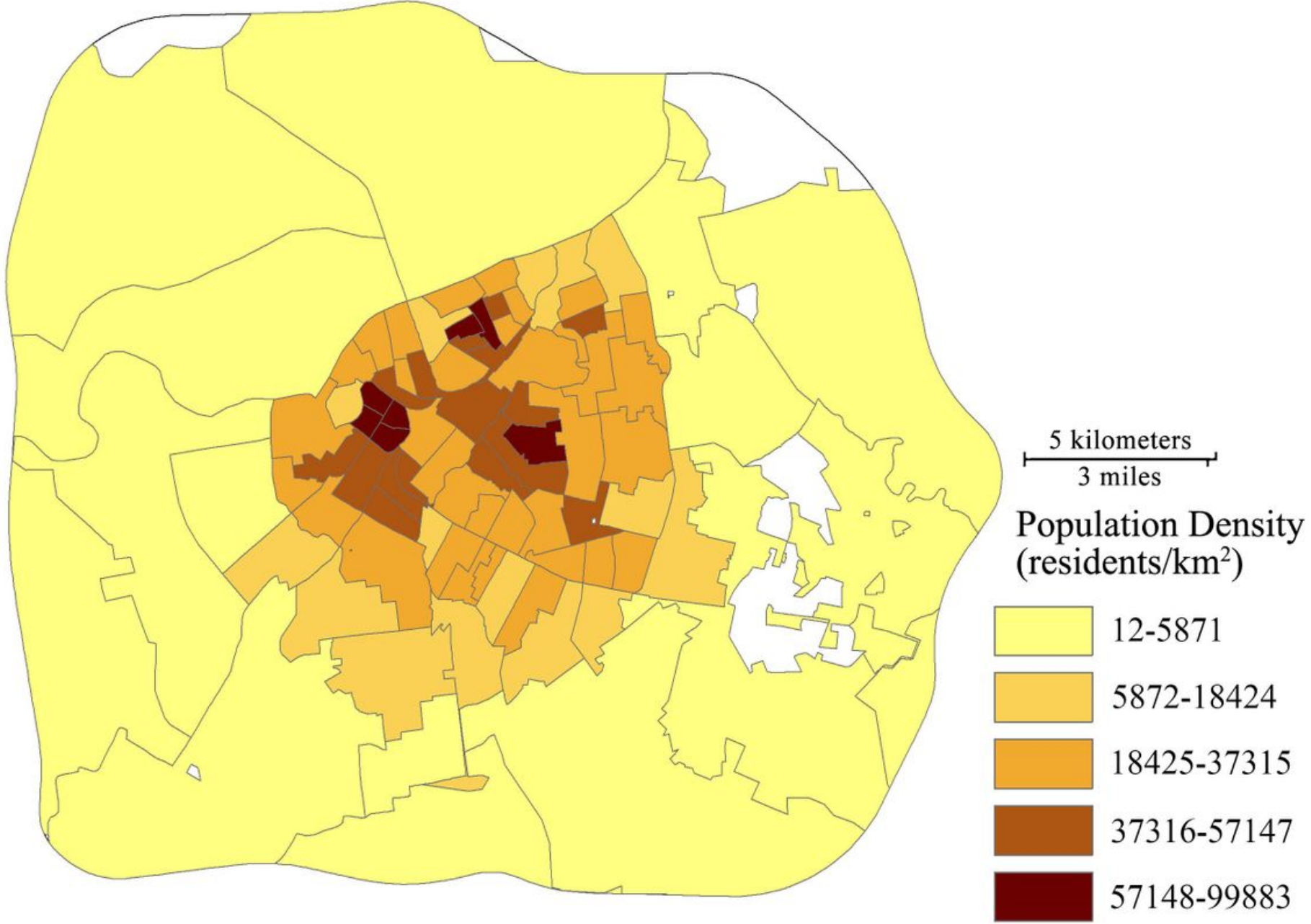


Figure 7

Spatial Distribution of Population Density in Harbin

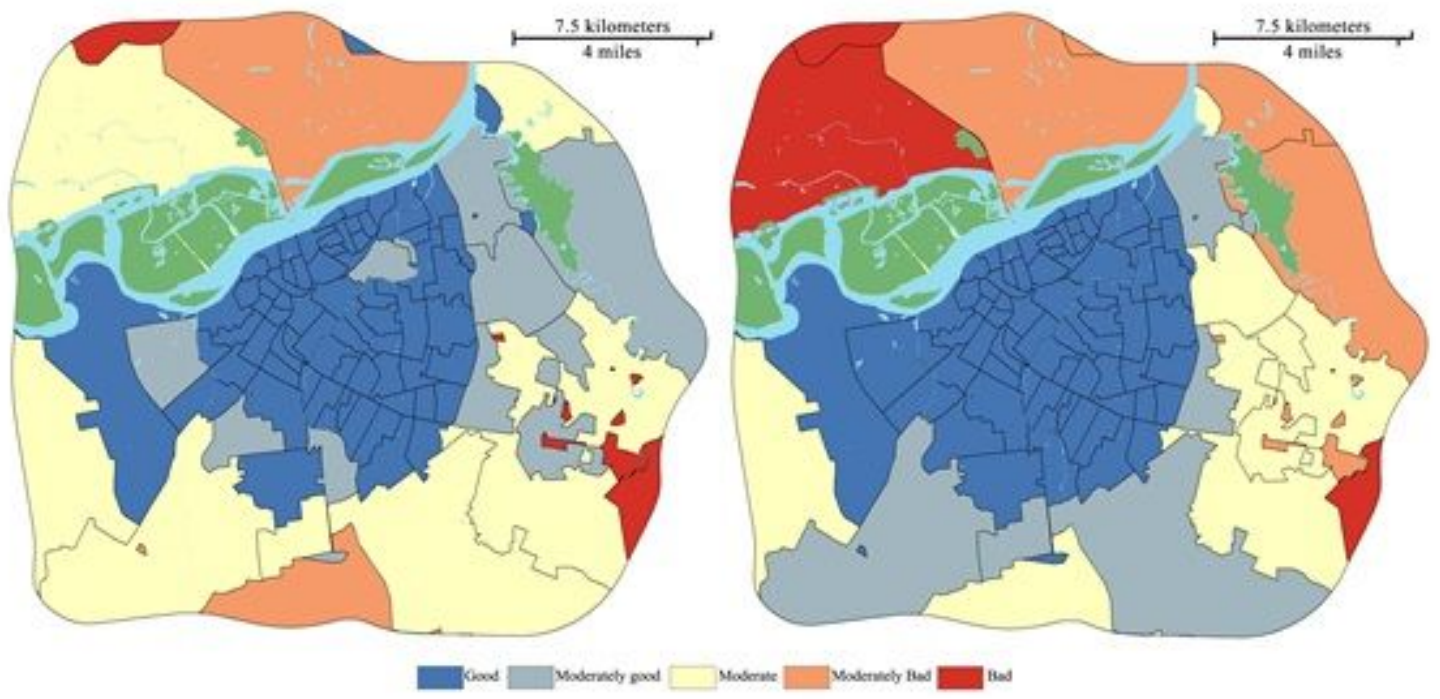

a Accessibility of Community Healthcare Centers

b Accessibility of General Hospitals

\section{Figure 8}

Accessibility of Medical Institutions in Harbin

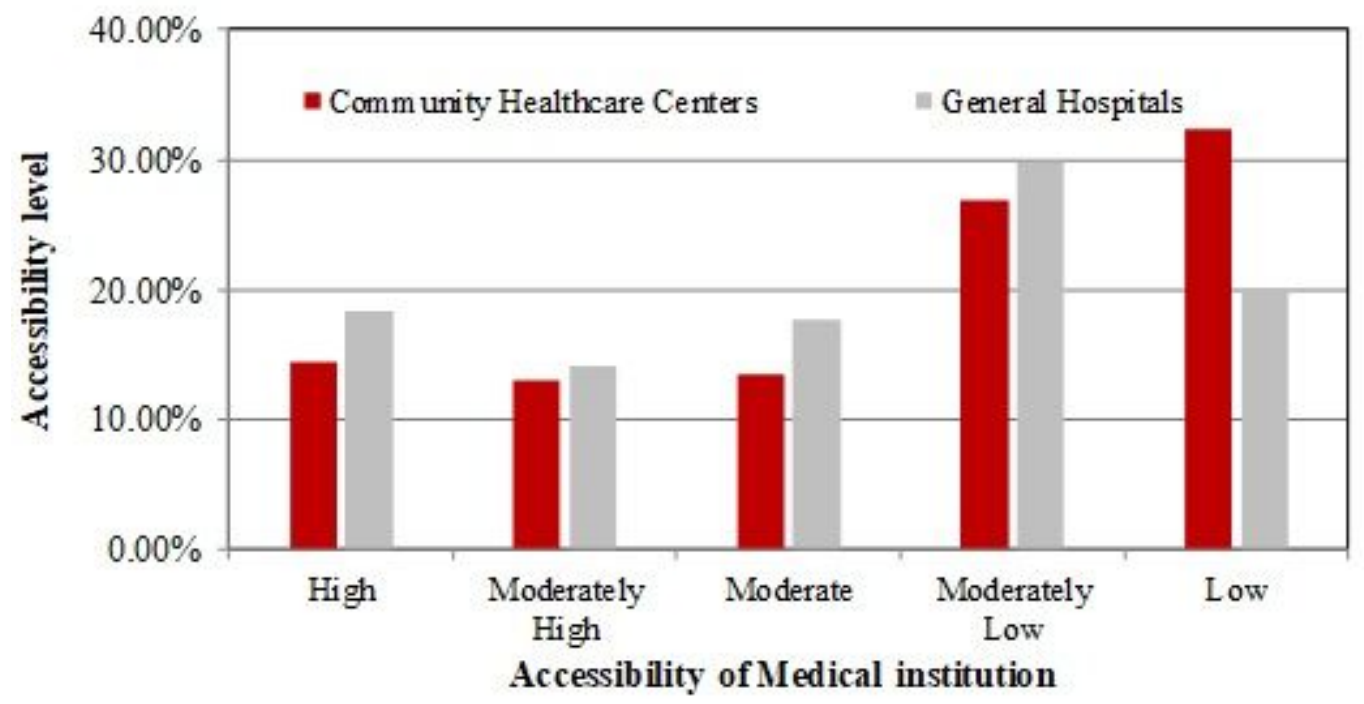

Figure 9

Accessibility of Medical Institutions in Harbin Based on a Weighted Spatial Separation Model

\section{Supplementary Files}

This is a list of supplementary files associated with this preprint. Click to download. 
- Equations.pdf

Page 20/20 